\title{
Caracterización metabólica de Diplodon chilensis (Gray, 1828) (Bivalvia: Hyriidae) expuesto a anoxia experimental
}

\author{
Metabolic characterization of Diplodon chilensis (Gray, 1828) (Bivalvia: Hyriidae) \\ exposed to experimental anoxia \\ Maritza A. Grandón ${ }^{1}$, Javier A. Barros ${ }^{1}$ y Rodrigo R. González ${ }^{1,2}$ \\ ${ }^{1}$ Ingeniería en Biotecnología Marina y Acuicultura, Facultad de Ciencias Naturales y Oceanográficas, \\ Universidad de Concepción, Casilla 160-C, Concepción, Chile \\ ${ }^{2}$ Centro de Investigación Oceanográfica en el Pacífico Sur-Oriental (COPAS), Universidad de Concepción, \\ Casilla 160-C, Concepción, Chile \\ maritzagrandon@udec.cl
}

\begin{abstract}
Diplodon chilensis is a freshwater bivalve inhabiting from northern Chile to Tierra del Fuego. This species has a high filtration capacity and great tolerance to hypoxic environments. Thus, the aim of this study was to characterize, for the first time, anaerobic metabolism of $D$. chilensis using an enzymatic approach by measuring piruvate oxidoreductases and malate dehydrogenase activities in two groups of organisms. A first group (G1) corresponded to organisms analyzed immediately after their collection from Lleu-Lleu Lake $\left(36^{\circ} \mathrm{S}\right)$. The second group (G2) corresponded to organisms analyzed after exposition to extreme anoxic conditions in laboratory. Enzymatic activities were measured in abductor muscle, foot, gills, hepatopancreas gland, and mantle-gonad tissue. Positive activities were detected for piruvate oxidoreductases (PORs: lactate dehydrogenase (LDH), octopine dehydrogenase $(\mathrm{OPDH})$, strombine dehydrogenase (STRDH), alanopine dehydrogenase (ALPDH)) and malate dehydrogenase (MDH). $\mathrm{MDH}$ showed the highest dehydrogenase activity observed in all tissue and groups analyzed. Nevertheless, only those organisms exposed to extreme anoxic conditions showed significant relationships between $\mathrm{MDH}$ and $\mathrm{LDH}$ activities and a high ratio of $(\mathrm{MDH} / \mathrm{LDH})$ between them. All these enzymatic features of $D$. chilensis correspond to a species with a successfully anaerobic metabolism to cope with extreme anaerobic conditions.
\end{abstract}

Key words: LDH, MDH, opine, metabolism

\section{Introducción}

Las almejas de agua dulce del género Diplodon se distribuyen sólo en el cono sur americano. Es el único grupo que habita los cuerpos de aguas continentales, generalmente en sistemas lénticos, aunque también es posible encontrarlo en los remansos de los ríos (Parada \& Peredo 1994). La especie Diplodon chilensis (Gray, 1828) se encuentra distribuida desde el norte de Chile hasta la Patagonia y Tierra del
Resumen.- Diplodon chilensis es un bivalvo dulceacuícola que habita desde el norte de Chile hasta Tierra del Fuego, posee una alta capacidad de filtración y gran tolerancia a ambientes hipóxicos. Por esta razón, el objetivo de este estudio fue caracterizar por primera vez el metabolismo anaeróbico de $D$. chilensis a través de la actividad enzimática de las piruvato oxidoreductasas y malato dehidrogenasa analizadas en dos grupos de organismos. El primer grupo (G1) correspondió a organismos analizados inmediatamente después de su recolección desde el lago Lleu-Lleu $\left(36^{\circ} \mathrm{S}\right)$ y el segundo grupo (G2) correspondió a organismos expuestos a condiciones experimentales extremas de anoxia. Se determinó la actividad enzimática involucrada en el catabolismo anaeróbico en cinco tejidos (músculo abductor, pie, branquias, hepatopáncreas y manto-gónada). Se detectó actividad positiva para las piruvato oxido reductasas (PORs: lactato dehidrogenasa (LDH), octopina dehidrogenasa (OPDH), strombina dehidrogenasa (STRDH), alanopina dehidrogenasa (ALPDH)) y malato dehidrogenasa (MDH). MDH mostró la mayor actividad dehidrogenasa observada en todos los tejidos en los dos grupos analizados. No obstante, solo en los organismos del grupo G2 se observó una correlación significativa entre las actividades de MDH y LDH, así como un mayor valor para la razón MDH/ LDH. Todas estas características enzimáticas determinadas en D. chilensis corresponden a una especie con un metabolismo anaeróbico que le permite enfrentar exitosamente condiciones anóxicas extremas.

Palabras clave: LDH, MDH, opinas, metabolismo

Fuego (Haas 1969, Peredo \& Parada 1984), y en Argentina desde la Provincia de Mendoza hasta la Patagonia chilenoargentina (Bonetto 1973).

Diplodon chilensis posee una gran capacidad para vivir en distintos ambientes dulceacuícolas (Parada 1987) alcanzando densidades variables y crecientes desde 10 ind. $\mathrm{m}^{-2}$ en sustratos arenosos a 361 ind. $\mathrm{m}^{-2}$ en sustratos fangosos (Lara \& Parada 1988). Diplodon constituye un 
componente importante del macrozoobentos, debido a su gran capacidad de filtración (Busse 1970, Valdovinos \& Cuevas 1996) y remoción de bacterias (Sepúlveda 1988) en ambientes hipóxicos y cuerpos de agua que reciben cargas significativas de materia orgánica (Busse 1970, Lara et al. 2002a, Soto \& Mena 1999). En especial, se ha determinado que este organismo influye sobre la ecología del fitoplancton (Daukas et al. 1981), la calidad de las aguas y el ciclaje de nutrientes (Soto \& Mena 1999), contribuyendo por esta vía a mantener la oligotrofia de los cuerpos acuáticos donde habitan (Lara et al. 2002b). Así también, se ha postulado que $D$. chilensis puede ser utilizado con fines biotecnológicos en el tratamiento de aguas servidas y biorremediación (Lara et al. 2002a, Sepúlveda 1988, Vallejos 1996). Estas características sugieren que $D$. chilensis debe tener un activo metabolismo anaeróbico para sobrevivir en ambientes con alta carga de materia orgánica y pobres en oxígeno.

Considerando las vías metabólicas anaeróbicas que participan en el catabolismo celular, la vía del lactato y las opinas (octopina, strombina y alanopina) han sido caracterizadas de manera general, como la actividad de las piruvato oxidoreductasas (PORs: lactato dehidrogenasa o LDH, octopina dehidrogenasa u OPDH, strombina dehidrogenasa o STRDH y alanopina dehidrogenasa o ALPDH) y definidas como las vías fermentativas en procariontes y metazoarios (Hochachka \& Somero 1984). En organismos acuáticos, la mayoría de los estudios sobre el metabolismo anaeróbico han estado centrados en la adaptación de los organismos bentónicos a condiciones hipóxicas. En estos organismos se ha establecido un desplazamiento hacia la presencia de la actividad de las opinas dehidrogenasas (OPDH, STRDH y ALPDH) por sobre la actividad de LDH, como la expresión de la actividad de las vías fermentativas (Livingstone 1983, 1991). Sin embargo, en los organismos pelágicos se ha observado un dominio de la actividad LDH sobre las opinas (Livingstone 1983, 1991, Grieshaber et al. 1994). En términos teóricos, la diferencia energética potencial entre el uso de la vía del lactato o las opinas está basada en el número y disponibilidad de substratos, y no en su rendimiento energético neto, lo que incidiría en el tipo de vía fermentativa utilizada por las especies (Livingstone 1983). No obstante, también se postula que a un mayor número de piruvato oxidoreductasas presentes en un organismo acuático, mayor es su capacidad para resistir los cambios en un gradiente de concentración de oxígeno (Grieshaber et al. 1994, González \& Quiñones 2000).

También, como elementos del catabolismo celular, la enzima malato dehidrogenasa (MDH) en eucariontes, está involucrada en varias reacciones del metabolismo energético (Lehninger 1975). Comparte la función de intercambio de electrones desde el citoplasma a la matriz mitocondrial vía reducción de oxalacetato a malato en presencia de NADH (Nicotidamín Adenín Dinucleotido, especie reducida) y viceversa, donde el malato en el interior de la matriz es oxidado nuevamente a oxalacetato en presencia de $\mathrm{NAD}^{+}$(especie oxidada). La regulación de este proceso es clave para mantener el balance redox intracelular y/o producción de energía al interior de la célula durante el metabolismo anaeróbico intenso (alta tasa catabólica), de manera similar a la captura de electrones en los productos de las vías fermentativas (Hochachka \& Somero 1984). Adicionalmente, esta condición sugiere la importancia de la actividad de la $\mathrm{MDH}$ en el metabolismo anaeróbico involucrado en las respuestas metabólicas requeridas para enfrentar un gradiente redox ambiental y/o fisiológico (Livingstone 1983). Así también, se ha postulado que mientras mayor sea la relación de la actividad de $\mathrm{MDH}$ con la $\mathrm{LDH}$, mayor es la capacidad del organismo para resistir la hipoxia ambiental (Shapiro \& Bobkova 1975).

Por lo anteriormente expuesto y considerando el tipo de ambiente y funciones asociadas a D. chilensis, hipotéticamente, esta especie debería poseer un alto metabolismo anaeróbico expresado como el mayor número de actividades enzimáticas de las piruvato oxidoreductasas (LDH, OPDH, STRDH y ALPDH) y una alta relación de las actividades $\mathrm{MDH} / \mathrm{LDH}$ cuando su metabolismo responde a condiciones de mínimo oxígeno, como por ejemplo en ambientes con una fuerte carga de materia orgánica. Por lo tanto, el objetivo de este trabajo fue 1) determinar la presencia de la actividad de piruvato oxidoreductasas y $\mathrm{MDH}$ en organismos tomados directamente desde el ambiente y 2) analizar la respuesta de la actividad de estas enzimas (respuesta metabólica) de $D$. chilensis cuando este organismo es sometido a condiciones de mínimo oxígeno y alta materia orgánica comparado con el medio en que habita.

\section{Material y métodos}

Los ejemplares de Diplodon chilensis fueron obtenidos desde el Lago Lleu-Lleu, situado a $14 \mathrm{~km}$ al suroeste de Contulmo ( $\left.38^{\circ} 07^{\prime} \mathrm{S}, 73^{\circ} 19^{\prime} \mathrm{O}\right)$, en el centro-sur de Chile, durante el verano de 2005. Los organismos fueron recolectados manualmente entre 0,5 y $2,5 \mathrm{~m}$ de profundidad mediante buceo apnea y correspondieron a una muestra poblacional. Se seleccionó una parte de estos organismos $(n=14)$, en una escala de tamaño (entre 4,9 y $6,6 \mathrm{~cm}$ de largo y entre 2,4 y 3,1 cm de ancho), para el análisis de la actividad enzimática inmediatamente después de su recolección. Se denominará operacionalmente en lo sucesivo a este grupo como G1. Estos organismos 
no fueron aclimatados antes de su análisis, debido a que la finalidad es conocer el perfil de actividad enzimática anaeróbica presente en $D$. chilensis en su condición natural. Estos especimenes fueron congelados casi instantáneamente en nitrógeno líquido, inmediatamente después de su recolección con el objeto de minimizar el efecto del estrés por transporte o almacenamiento. Los organismos restantes $(n=33)$ fueron utilizados para realizar el experimento de eutrofización y serán denominados operacionalmente en lo sucesivo como G2. Previo al experimento, los individuos fueron mantenidos en contenedores de $5 \mathrm{~L}$ durante dos semanas con una densidad aproximada de 3 ind. $\mathrm{L}^{-1}$ de agua y en condiciones saturantes de oxígeno (aireación constante), alimentados con una dieta de levadura y recambio del $50 \%$ de agua cada dos días. El experimento se inició al interrumpir el suministro de oxígeno e incrementar la densidad de $D$. chilensis al doble por litro de agua, continuándose con la alimentación basada en levadura y sin recambio de agua durante un mes. Esto produjo la eutrofización total del acuario registrando una mortalidad aproximadamente de un $20 \%$, los organismos sobrevivientes $(\mathrm{n}=26)$ fueron analizados. La concentración de oxígeno disuelto fue estimada utilizando un micro-método semiautomatizado basado en el método de Winkler (Williams \& Jenkinson 1982). El oxígeno en el acuario se agotó a los dos días del ensayo (no detectable) y permaneció así hasta el final del experimento. Todos los organismos fueron mantenidos a la misma temperatura $\left( \pm 15^{\circ} \mathrm{C}\right) \mathrm{e}$ igual fotoperiodo (12 h luz: $12 \mathrm{~h}$ oscuridad).

En todos los organismos (G1 y G2), se analizaron los siguientes tejidos: músculo abductor, pie, branquias, hepatopáncreas y manto-gónada. Se registraron, además, el largo y ancho de la concha, peso húmedo total y de cada tejido.

Las enzimas analizadas fueron: piruvato oxidoreductasas (PORs): lactato dehidrogenasa (LDH), octopina dehidrogenasa (OPDH), alanopina dehidrogenasa (ALPDH) y strombina dehidrogenasa (STRDH). Además, también se analizó la actividad de malato dehidrogenasa (MDH). Todas las actividades están expresadas como actividades específicas aparentes en $\mu \mathrm{mol} \mathrm{NADH}$ min $^{-1} \mathrm{~g}^{-1}$ peso húmedo (UI g-1 peso húmedo).

El tampón de homogenización fue de $400 \mathrm{mM}$ tampón fosfato $\left(\mathrm{K}_{2} \mathrm{HPO}_{4}\right), \mathrm{pH} 7,9,0,3 \%$ (peso/vol) polivinil pirrolidona (PVP), 5mM EDTA, 0,1\% (vol/vol) Triton $\mathrm{X}-100$. Las muestras fueron homogenizadas en un UltraTurrax con una proporción 1:10 peso/volumen durante 30 s, a máxima velocidad, sobre un baño de hielo. Los homogenizados fueron centrifugados a $3000 \mathrm{~g}$ x 5 minutos a $4^{\circ} \mathrm{C}$. Las actividades enzimáticas fueron determinadas en los sobrenadantes de la centrifugación anterior.

Las actividades de las PORs (LDH, OPDH, ALPDH y STRDH) fueron medidas utilizando una mezcla de reacción modificada desde el método de Schiedek (1997) que contenía tampón $400 \mathrm{mM} \mathrm{K}_{2} \mathrm{HPO}_{4} \mathrm{pH} 7,9$ a $20^{\circ} \mathrm{C}$, 2,5 mM NADH. Dependiendo de la actividad a determinar, se agregaron 3,2 $\mathrm{mM}$ de piruvato para $\mathrm{LDH}$, $3,2 \mathrm{mM}$ piruvato y 2,7 $\mathrm{mM}$ arginina para $\mathrm{OPDH}, 3,2 \mathrm{mM}$ piruvato y $250 \mathrm{mM}$ de alanina para ALPDH, o 3,2 $\mathrm{mM}$ piruvato y $250 \mathrm{mM}$ glicina para STRDH. Las reacciones se iniciaron con la adición de una alícuota de sobrenadante y se midió el decaimiento de la absorción de NADH a $340 \mathrm{~nm}$. Todas las actividades enzimáticas fueron corregidas por la oxidación inespecífica de NADH y las actividades de OPDH, ALPDH y STRDH también fueron corregidas por la actividad de LDH, ambos métodos de corrección según Schiedek (1997).

La actividad de malato dehidrogenasa (MDH) fue determinada utilizando el método modificado descrito por Childress \& Somero (1979) y Vetter et al. (1994) en el sentido de la reducción de oxalacetato. La mezcla de reacción contenía $0,1 \mathrm{mM}$ de $\mathrm{NADH}, 1,5 \mathrm{mM} \mathrm{MgSO}_{4} \mathrm{y}$ $0,2 \mathrm{mM}$ oxalacetato. Las reacciones se iniciaron con la adición de una alícuota de sobrenadante y se midió el decaimiento de la absorción de NADH a $340 \mathrm{~nm}$. La actividad de $\mathrm{MDH}$ fue corregida por la oxidación inespecífica de NADH.

Para la verificación de diferencias significativas entre actividades enzimáticas de G1 y G2 se realizó una prueba de Wilcoxon, excepto en la comparación de las actividades promedio totales de $\mathrm{MDH}(\mathrm{n}=59$ para $\mathrm{G} 1$; $\mathrm{n}=71$ para $\mathrm{G} 2$ ) que fue realizada mediante una prueba $t$. Se realizó un análisis de regresión lineal entre las variables de las actividades de MDH y LDH (Zar 1996). Los análisis estadísticos fueron realizados a través del software Statistica 6.0.

\section{Resultados}

Tanto para G1 como G2 los resultados indican la existencia de actividad para todas las enzimas ensayadas (Figs. 1 y 2, Tabla 1), siendo MDH la mayor actividad determinada en todos los tejidos para ambos grupos. No obstante, la actividad de MDH resultó ser dos órdenes de magnitud mayor para los organismos del grupo G2 que para los organismos del grupo $\mathrm{G} 1(\mathrm{~F}=22,31 ; P<0,001)$. Para esta actividad, los organismos del grupo G2 no mostraron diferencias significativas entre los tejidos, no obstante, la actividad de MDH en el pie de los organismos del grupo G1 fue significativamente mayor a todos los tejidos analizados (músculo abductor: $\mathrm{Z}=2,12, P=0,030$; 


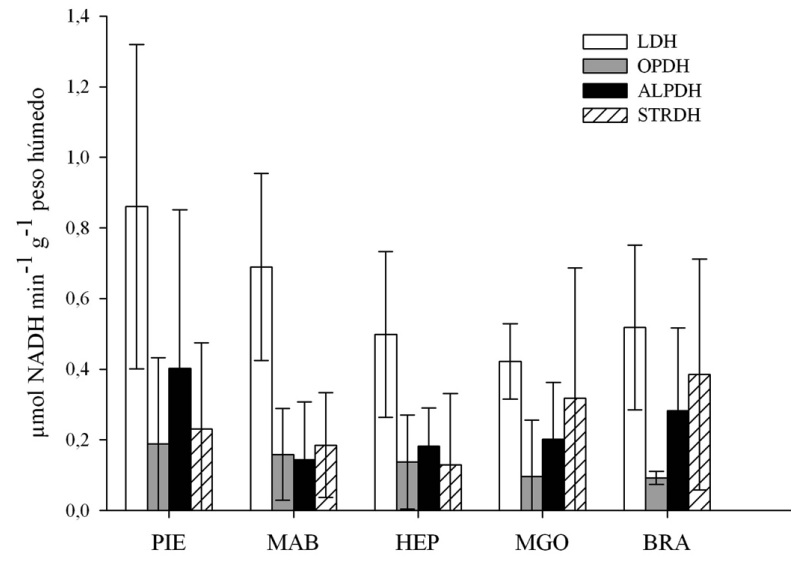

Figura 1

Actividad enzimática promedio \pm desviación estandar (d.e) por tejido ( $\mu$ mol NADH $\mathrm{min}^{-1} \mathrm{~g}^{-1}$ peso húmedo) de piruvato oxidoreductasas (PORs: $L D H=$ lactato dehidrogenasa, OPDH = octopina dehidrogenasa, STRDH = strombina dehidrogenasa y ALPDH = alanopina dehidrogenasa), determinadas en organismos del grupo G1 (analizados inmediatamente después de su recolección). Abreviaciones del tejido del bivalvo en la Tabla 1

Average \pm standard deviation (s.d.) of enzymatic activity

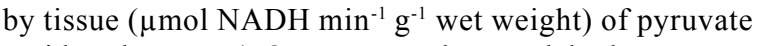
oxidoreductases (PORs: $\mathrm{LDH}=$ lactate dehydrogenase, $\mathrm{OPDH}=$ octopine dehydrogenase, STRDH = strombine dehydrogenase and ALPDH = alanopine dehydrogenase), measured in organisms of G1 group (corresponding to organisms analyzed immediately after their recollection).

Abbreviations of the bivalve tissues are in Table 1

manto-gónada: $\mathrm{Z}=2,43, P=0,020$; branquias: $\mathrm{Z}=3,06$, $P=0,002)$ excepto con el hepatopáncreas $(Z=1,49 ; P=$ 0,14) (Fig. 1). En promedio, la actividad de LDH fue la mayor actividad de PORs en todos los tejidos y organismos analizados excepto en pie y en las branquias en los organismos del grupo G1 donde no hubo diferencias significativas con la actividad de ALPDH (pie: $Z=0,94$, $P=0,34$; branquias: $\mathrm{Z}=1,01, P=0,31)$ y en músculo abductor en los organismos del grupo G2 donde no se encontraron diferencias significativas al igual que con la actividad de ALPDH ( $\mathrm{Z}=1,60, P=0,11)$ (Fig. 2). La actividad de las opinas dehidrogenasas fue similar en casi todos los tejidos y organismos analizados, excepto para OPDH cuya actividad fue significativamente mayor $(\mathrm{Z}=$ $2,36, P=0,02)$ en el pie de los organismos del grupo G2 (Fig. 2). Considerando la actividad de todos los tejidos se observó una correlación positiva y significativa entre la actividad de MDH y LDH para los organismos del grupo G2 (Fig. 3), lo cual no se observó en los organismos del grupo G1.

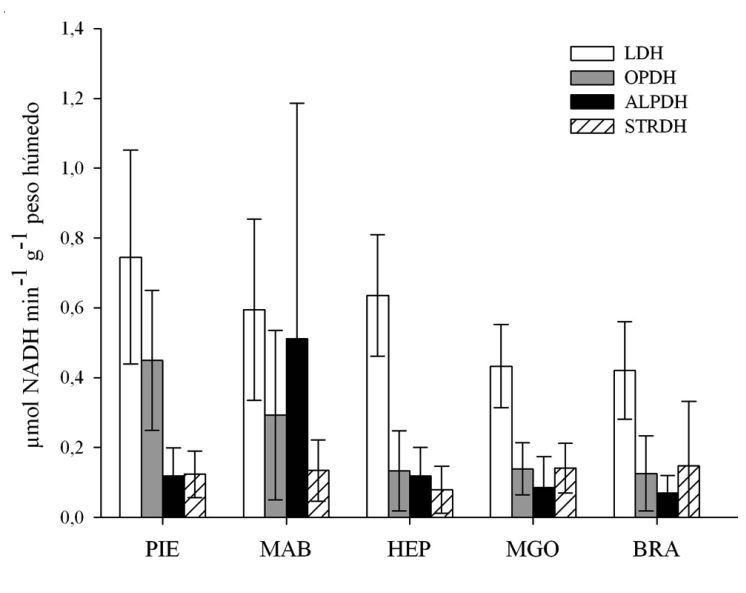

Figura 2

Actividad enzimática promedio \pm d.e. por tejido $(\mu \mathrm{mol}$ NADH min $^{-1} \mathrm{~g}^{-1}$ peso húmedo) de piruvato oxidoreductasas (PORs: $\mathrm{LDH}=$ lactato dehidrogenasa, $\mathrm{OPDH}=$ octopina dehidrogenasa, STRDH = strombina dehidrogenasa y ALPDH = alanopina dehidrogenasa), determinadas en organismos del grupo G2 (organismos expuestos a anoxia extrema durante un mes)

Average \pm s.d. of enzymatic activity by tissue ( $\mu$ mol NADH $\min ^{-1} \mathrm{~g}^{-1}$ wet weight) of pyruvate oxidoreductases (PORs: $\mathrm{LDH}=$ lactate dehydrogenase, $\mathrm{OPDH}=$ octopine dehydrogenase, STRDH = strombine dehydrogenase and ALPDH = alanopine dehydrogenase), measured in organisms of G2 group (organisms exposed to extreme anoxic conditions during a month)

\section{Discusión}

La determinación de la presencia de actividad enzimática de las cuatro PORs ensayadas indica que D. chilensis posee una gran capacidad metabólica para resistir condiciones anóxicas, siendo consistente con el planteamiento general de que a un mayor número de estas enzimas en un organismo acuático, mayor es su capacidad metabólica para enfrentar condiciones de mínimo oxígeno (Grieshaber et al. 1994, González \& Quiñones 2000). Es muy difícil comparar los valores obtenidos para las actividades enzimáticas en este estudio con otros bivalvos de agua dulce, debido al escaso número de trabajos relacionados con esta temática. Sin embargo, los valores aquí reportados para LDH están en el mismo rango a los informados para el molusco de agua dulce Melanoides tuberculata (Wepener et al. 2005) y con respecto a bivalvos marinos, las actividades de las PORs y MDH están en el mismo rango a los encontrados en Calyptogena gallardoi (González et al. 2008), recientemente descrita como un organismo que habita zonas de mínimo oxígeno alrededor de emanaciones frías de metano en el Sistema de la Corriente de Humboldt (Sellanes \& Krylova 2005). 
Tabla 1

Actividad enzimática promedio \pm desviación estándar (d.e.) por tejido ( $\mu$ mol NADH minn $^{-1} \mathrm{~g}^{-1}$ peso húmedo) de malato dehidrogenasa (MDH) y piruvato oxidoreductasas (PORs: LDH = lactato dehidrogenasa, OPDH = octopina dehidrogenasa, STRDH = strombina dehidrogenasa y ALPDH = alanopina dehidrogenasa), para los organismos del grupo G1 (organismos analizados inmediatamente después de su recolección) y el grupo G2 (organismos expuestos a anoxia extrema durante un mes). Las diferencias significativas $(P<0,05)$ están indicadas por * para la actividad de MDH para ambos grupos

Average \pm s.d. of enzymatic activity by tissue ( $\mu$ mol NADH $\min ^{-1} \mathrm{~g}^{-1}$ wet weight) of malate dehydrogenase (MDH) and pyruvate oxidoreductases (PORs: $\mathrm{LDH}=$ lactate dehydrogenase, $\mathrm{OPDH}=$ octopine dehydrogenase, $\mathrm{STRDH}=$ strombine dehydrogenase and ALPDH = alanopine dehydrogenase), measured in organisms of G1 (organisms analyzed immediately after being collected) and G2 group (organisms exposed to extreme anoxia during a month). Significant differences $(P<0.05)$ are indicated by * for MDH activities for both groups

\begin{tabular}{lcccccc}
\hline & & \multicolumn{5}{c}{ Enzima } \\
\cline { 3 - 7 } \multicolumn{1}{c}{ Tejido } & Grupo & MDH & LDH & OPDH & ALPDH & STRDH \\
\hline Pie (PIE) & G1 & $163,3 \pm 53,4^{*}$ & $0,745 \pm 0,306$ & $0,449 \pm 0,201$ & $0,124 \pm 0,067$ & $0,119 \pm 0,081$ \\
& G2 & $(38,5 \pm 12,2)$ & $(0,86 \pm 0,46)$ & $(0,19 \pm 0,24)$ & $(0,40 \pm 0,45)$ & $(0,23 \pm 0,24)$ \\
Músculo abductor (MAB) & G1 & $159,0 \pm 59,1^{*}$ & $0,595 \pm 0,259$ & $0,293 \pm 0,242$ & $0,134 \pm 0,088$ & $0,512 \pm 0,674$ \\
& G2 & $(25,6 \pm 11,2)$ & $(0,69 \pm 0,26)$ & $(0,16 \pm 0,13)$ & $(0,14 \pm 0,16)$ & $(0,19 \pm 0,15)$ \\
Hepatopáncreas (HEP) & G1 & $166,0 \pm 56,3^{*}$ & $0,635 \pm 0,174$ & $0,134 \pm 0,114$ & $0,079 \pm 0,067$ & $0,119 \pm 0,081$ \\
& G2 & $(29,0 \pm 13,8)$ & $(0,50 \pm 0,23)$ & $(0,14 \pm 0,13)$ & $(0,18 \pm 0,11)$ & $(0,13 \pm 0,20)$ \\
Branquias (BRA) & G1 & $133,3 \pm 53,4^{*}$ & $0,421 \pm 0,139$ & $0,126 \pm 0,108$ & $0,148 \pm 0,184$ & $0,071 \pm 0,050$ \\
& G2 & $(26,1 \pm 10,5)$ & $(0,42 \pm 0,11)$ & $(0,10 \pm 0,16)$ & $(0,20 \pm 0,16)$ & $(0,32 \pm 0,37)$ \\
Manto-Gónada (MGO) & G1 & $146,1 \pm 60,5^{*}$ & $0,433 \pm 0,120$ & $0,139 \pm 0,071$ & $0,141 \pm 0,071$ & $0,086 \pm 0,088$ \\
& G2 & $(22,8 \pm 9,5)$ & $(0,52 \pm 0,23)$ & $(0,09 \pm 0,02)$ & $(0,28 \pm 0,23)$ & $(0,39 \pm 0,33)$ \\
\hline
\end{tabular}

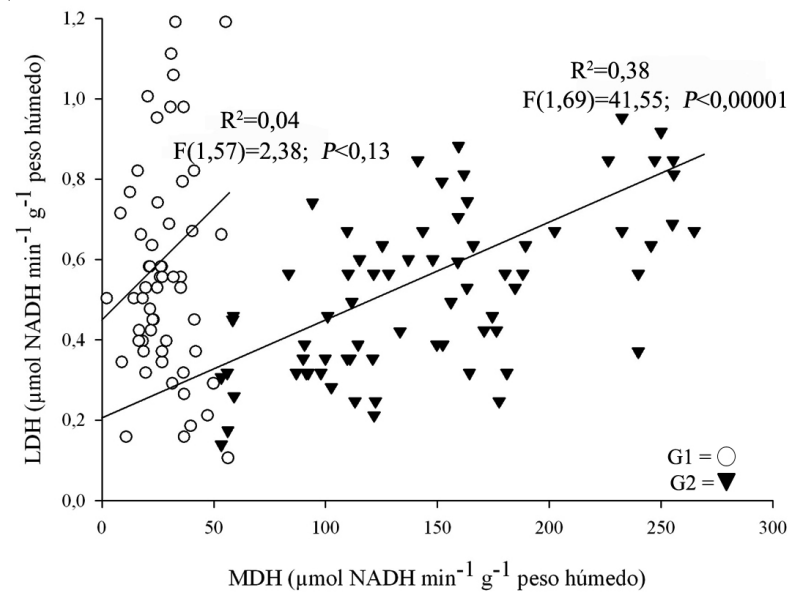

Figura 3

Correlación entre las actividades enzimáticas de malato dehidrogenasa (MDH) y lactato dehidrogenasa (LDH) determinadas en organismos del grupo G1 (analizados inmediatamente después de su recolección) y G2 (organismos expuestos a anoxia extrema durante un mes)

Correlation between the enzymatic activities of malate dehydrogenase (MDH) and lactate dehydrogenase

(LDH) determined in organisms of G1 group

(organisms analyzed immediately after their recollection) and $\mathrm{G} 2$ group organisms exposed to extreme anoxic conditions during a month)
Los resultados indican que la condición de anoxia extrema induce una respuesta selectiva sobre el metabolismo anaeróbico en $D$. chilensis. Esta respuesta se caracteriza por el incremento en la actividad de MDH, lo que fue determinado en todos los tejidos de los organismos del grupo G2. Este incremento es consistente con el aumento de actividad de MDH descrito durante el metabolismo anaeróbico intenso (Hochachka \& Somero 1984). El aumento en la actividad de MDH también incrementa el valor de la relación $\mathrm{MDH} / \mathrm{LDH}$ (Tabla 2) para el grupo G2 en comparación con G1. En invertebrados se ha demostrado que cuando el valor de esta relación se hace mucho mayor a uno, mejor es la capacidad del organismo para tolerar las condiciones anóxicas del medio (Shapiro \& Bobkova 1975). No obstante, en $D$. chilensis la razón de $\mathrm{MDH}$ con cualquiera otra de las PORs ensayadas también fue mucho mayor a uno, lo que en conjunto con el gran número de PORs detectadas es una fuerte evidencia de que esta especie posee una maquinaria bioquímica que le permitiría potencialmente sobrevivir al $80 \%$ de la población frente a una condición anóxica del medio, al menos durante el periodo que comprendió el presente estudio (un mes). Más aún, los resultados indican que la actividad de MDH y LDH en los organismos del grupo G1 no poseen una correlación (Fig. 3), lo que implicaría que ambas reacciones no estarían metabólicamente actuando coordinadamente en un ambiente saturado de oxígeno. En contraste, en el grupo G2, la exposición de D. chilensis 
Tabla 2

Razón promedio \pm d.e. entre la actividad de malato dehidrogenasa (MDH) y piruvato oxidoreductasas (abreviaciones en Tabla 1), determinadas en organismos del grupo G1 y G2

Mean rate \pm s.d. between the activities of malate dehydrogenase (MDH) and pyruvate oxidoreductases (abbreviations on Table 1), measured in organisms of G1 and G2 groups

\begin{tabular}{lccccc}
\hline & & \multicolumn{4}{c}{ Actividad MDH/PORs } \\
\cline { 3 - 6 } \multicolumn{1}{c}{ Tejido } & Grupo & MDH/LDH & MDH/OPDH & MDH/ALPDH & MDH/STRDH \\
\hline Pie & G1 & $66,9 \pm 61,4$ & $27,9 \pm 247,3$ & $65,5 \pm 58,7$ & $70,2 \pm 60,3$ \\
& G2 & $(197,6 \pm 162,1)$ & $(149,9 \pm 171,7)$ & $(216,0 \pm 169,3)$ & $(201,5 \pm 178,0)$ \\
Músculo abductor & G1 & $42,3 \pm 28,7$ & $58,4 \pm 38,3$ & $75,1 \pm 57,1$ & $49,4 \pm 83,4$ \\
& G2 & $(277,2 \pm 111,8)$ & $(205,8 \pm 105,2)$ & $(351,8 \pm 225,1)$ & $(280,4 \pm 130,4)$ \\
Hepatopáncreas & G1 & $103,9 \pm 146,4$ & $80,0 \pm 46,7$ & $141,5 \pm 308,5$ & $133,7 \pm 294,8$ \\
& G2 & $(251,1 \pm 99,9)$ & $(372,2 \pm 566,6)$ & $(345,7 \pm 253,6)$ & $(345,8 \pm 355,8)$ \\
Branquias & G1 & $51,1 \pm 28,6$ & $72,5 \pm 32,2$ & $74,2 \pm 77,1$ & $73,1 \pm 69,0$ \\
& G2 & $(333,7 \pm 148,6)$ & $(297,9 \pm 119,1)$ & $(319,4 \pm 128,2)$ & $(297,7 \pm 121,7)$ \\
Manto-Gónada & G1 & $73,4 \pm 57,8$ & $27,7 \pm 134,2$ & $59,6 \pm 25,3$ & $82,2 \pm 102,0$ \\
& G2 & $(350,1 \pm 145,1)$ & $(290,4 \pm 81,2)$ & $(367,4 \pm 160,8)$ & $(296,0 \pm 128,4)$ \\
\hline
\end{tabular}

a condiciones extremas de anoxia induce una correlación positiva y significativa en las actividades de MDH y LDH en el $80 \%$ de los sobrevivientes.

Si bien los resultados obtenidos en este estudio corresponden a una respuesta metabólica a corto plazo cuando esta especie es sometida a condiciones extremas de anoxia, la caracterización de la actividad enzimática obtenida en los organismos del grupo G1 corresponde a la condición en estado natural de su metabolismo anaeróbico y representa su estrategia metabólica adaptativa potencial frente a la disminución de oxígeno del medio. Además estos resultados permiten postular que la correlación encontrada entre las actividades de MDH y LDH podría ser utilizada como un índice de condición metabólico, puesto que es sólo significativa cuando los organismos están enfrentados a un estrés ambiental (grupo G2) por falta de oxígeno y exceso de materia orgánica. Este planteamiento podría ser puesto a prueba determinando la actividad de estas enzimas en cuerpos de agua dulce diferentes al lago Lleu-Lleu, el que posee normalmente altas concentraciones de oxígeno $\left(9 \mathrm{mg} \mathrm{L}^{-1}\right.$; Parada \& Peredo 1994) y ha sido clasificado como en condiciones más prístinas que el vecino lago Lanalhue y otros lagos nahuelbutenses.

En conclusión, los resultados de las actividades enzimáticas determinadas en $D$. chilensis son consistentes con un organismo capaz de enfrentar metabólicamente una condición ambiental de mínimo oxígeno.

\section{Agradecimientos}

Los autores agradecen la colaboración de María Cristina Krautz en la recolección de los ejemplares, a Lilian Muñoz por su asistencia en laboratorio y a José Marileo por su asistencia operacional en la estación de Biología Marina sede Dichato (Universidad de Concepción), Concepción, Chile. Finalmente los autores desean agradecer a los tres evaluadores anónimos por sus valiosos comentarios y sugerencias que sin duda permitieron generar una impronta al manuscrito original. Este estudio fue financiado por el proyecto FONDECYT 1040534.

\section{Literatura citada}

Bonetto A. 1973. Náyades de la Patagonia. Revista de la Asociación de Ciencias Naturales del Litoral 4: 177-185.

Busse K. 1970. Nuevo método para medir flujos de agua producidos por organismos filtradores. Medición experimental en Diplodon chilensis (Gray), 1854 (Mollusca, Lamellibranchiata). Noticiario Mensual del Museo de Historia Natural 172: 3-10.

Childress J \& G Somero. 1979. Depth-related enzymatic activities in muscle, brain and heart of deep-living pelagic marine teleosts. Marine Biology 52: 273-283.

Daukas P, B Peterson \& W Bowden. 1981. The clearance rates of chlorophyll a of the freshwater mussels Anodonta implicata Say and Elliptio complanata (Lightfoot). In: Alkon D \& M O'rand (eds), The Biological Bulletin 161: 303-355. 
González R \& R Quiñones. 2000. Pyruvate oxidoreductases involved in glycolytic anaerobic metabolism of Polychaetes from the continental shelf off Central-South Chile. Estuarine, Coastal and Shelf Science 51: 507-519.

González R, R Quiñones, E Quiroga \& J Sellanes. 2008. Aerobic and anaerobic enzymatic activities of Calyptogena gallardoi (Vesicomiydae): a clam associated with methane cold seeps off Chile. Journal of the Marine Biological Association 88: 983-986.

Grieshaber M, I Hardewig, U Kreutzer \& H Pörtner. 1994. Physiological and metabolic responses to hypoxia in invertebrates. Reviews of Physiology, Biochemistry \& Pharmacology 125: 43-147.

Haas F. 1969. Superfamilia Unionacea, 633 pp. Das Tierreich, Berlin.

Hochachka P \& G Somero. 1984. Biochemical Adaptation, 538pp. Princeton University Press, Princeton.

Lara G \& E Parada. 1988. Distribución espacial y densidad de Diplodon chilensis chilensis (Gray, 1928) (Mollusca: Bivalvia) en el Lago Villarrica. Boletín de la Sociedad de Biología de Concepción 58: 105-114.

Lara G, A Contreras \& F Encina. 2002a. La almeja de agua dulce Diplodon chilensis (Bivalvia: Hyriidae) potencial biofiltro para disminuir los niveles de coliformes en pozos: Experimento de laboratorio. Gayana 66: 113-118.

Lara G, E Parada \& S Peredo. 2002b. Alimentación y conducta alimentaria de la almeja de agua dulce Diplodon chilensis (Bivalvia: Hyriidae). Gayana 66: 107-112.

Lehninger A. 1975. Biochemistry, 1104 pp. Worth Publishers, Nueva York.

Livingstone D. 1983. Invertebrate and vertebrate pathway of anaerobic metabolism: evolutionary considerations. Journal of the Geological Society 140: 27-37.

Livingstone D. 1991. Origins and evolution of pathways of anaerobic metabolism in the animal kingdom. American Zoologist 31: 522-534.

Parada E. 1987. Estrategias del ciclo vital de Diplodon chilensis chilensis (Gray, 1828) (Bivalvia: Hyriidae). Tesis para optar al grado de Magíster en Ciencias mención Zoología, Universidad Austral de Chile, Valdivia, 175 pp.

Parada E \& S Peredo. 1994. Un enfoque ecológico evolutivo de las estrategias de historia de vida de los híridos chilenos (Mollusca, Bivalvia). Boletín de la Sociedad de Biología de Concepción 65: 71-80.

Peredo S \& E Parada. 1984. Gonadal organization and gametogenesis in the fresh-water mussel Diplodon chilensis chilensis (Mollusca: Bivalvia). The Veliger 27(2): 126-133.
Schiedek D. 1997. Marenzelleria viridis (Verril, 1873) (Polychaeta), a new benthic species within European costal waters. Some metabolic features. Journal of Experimental Marine Biology and Ecology 211: 85-101.

Sellanes J \& E Krylova. 2005. A new species of Calyptogena (Bivalvia: Vesicomyidae) from a recently discovered methane seepage area off Concepción Bay, Chile $\left(36^{\circ} \mathrm{S}\right)$. Journal of the Marine Biological Association of the United Kingdom 85: 969-976.

Sepúlveda A. 1988. Estudio preliminar de la contaminación fecal de las aguas naturales utilizando un organismo bioindicador y la técnica de filtración de membrana. Seminario de titulación de Tecnología Médica, Universidad Austral de Chile, Valdivia, $50 \mathrm{pp}$.

Shapiro A \& A Bobkova. 1975. The role of malate dehidrogenase in adaptation to hypoxia in invertebrates. Journal of Evolutionary Biochemistry and Physiology 11: 478-479.

Soto D \& G Mena. 1999. Filter feeding by the freshwater mussel, Diplodon chilensis, as a biocontrol of salmon farming eutrophication. Aquaculture 171: 65-81.

Valdovinos C \& R Cuevas. 1996. Tasas de aclarancia de Diplodon chilensis (Bivalvia: Hyriidae): un suspensívoro bentónico dulceacuícola de Chile Central. Medio Ambiente 13(1): 114-118.

Vallejos P. 1996. Utilización de bivalvos de agua dulce (Diplodon chilensis) para tratamientos de aguas domésticas domiciliarias, 95pp. Informe Técnico, CIDERE Bío-Bío, Concepción.

Vetter R, E Lynn, M Garza \& A Costa. 1994. Depth zonation and metabolic adaptation in Dover sole Microstomus pacificus, and other deep-living flatfishes: factors that affect the sole. Marine Biology 120: 145-159.

Wepener V, J Vuren, F Chatiza, Z Mbizi, L Slabbert \& B Masola. 2005. Active biomonitoring in freshwater environments: early warning signals from biomarkers in assessing biological effects of diffusesources of pollutants. Physics and Chemistry of the Earth 30: 751-761.

Williams $\mathbf{P} \& \mathbf{N}$ Jenkinson. 1982. A transportable microprocessor controlled precise Winkler titration suitable for field station and shipboard use. Limnology and Oceanography 27: 576-584.

Zar JH. 1996. Biostatistical analysis, 276 pp. Prentice Hall, Englewood Cliffs. 